\title{
Avaliação dos fluxos e serviços da Rede de Atenção Odontológica de Fortaleza, Ceará
}

\author{
Evaluation of flows and services of the Dentistry Care Network of Fortaleza, Ceará \\ Evaluación de flujos y servicios de la Red de Atención Dental de Fortaleza, Ceará
}

Recebido: 23/01/2022 | Revisado: 01/02/2022 | Aceito: 09/02/2022 | Publicado: 13/02/2022

\author{
Andréia de Queiroz Nobre \\ ORCID: https://orcid.org/0000-0002-3014-2486 \\ Universidade Estadual do Ceará, Brasil \\ E-mail: andreiaorthomax@hotmail.com \\ Indara Cavalcante Bezerra \\ ORCID: https://orcid.org/0000-0003-0647-2490 \\ Universidade Estadual do Ceará, Brasil \\ E-mail: indaracavalcante@yahoo.com.br
}

\begin{abstract}
Resumo
A implantação da Política Nacional de Saúde Bucal tencionou organizar as redes de atenção no Sistema Único de Saúde. A investigação objetiva avaliar o fluxo de serviços odontológicos no município de Fortaleza, Ceará, nordeste do Brasil. Trata-se de uma pesquisa qualitativa, que utilizou como cenário as unidades de Atenção Primária em Saúde, Secundária (Centro de Especialidades Odontológicos) e Terciária do município. Participaram do estudo 04 gerentes e 09 dentistas das unidades selecionadas. A coleta de informações, realizada durante o ano de 2021, foi extraída por meio de uma entrevista. Utilizou-se a narrativa hermenêutica para análise dos discursos, dividindo-as em: acesso aos serviços odontológicos, a regulação dos serviços odontológicos e distribuição dos profissionais na rede. Verificou-se que as unidades apresentam áreas territoriais de grande dimensão que gera barreiras geográficas para se ter acesso aos serviços oferecidos. Esse é um dos fatores que gera filas de espera por procedimentos, ocasionando um absenteísmo significativo na oferta de serviços. Também identificado um número de profissionais insuficientes para atendimento da demanda e falhas na marcação de consultas. Concluiu-se que é necessário criar um protocolo padronizado de marcação de consultas na atenção primária, ampliar o quadro de profissionais de saúde bucal nesse nível de atenção e nos centros de especialidades odontológicas, bem como promover a implantação de novos centros.
\end{abstract}

Palavras-chave: Saúde bucal; Acesso aos serviço de saúde; Agendamento de consultas.

\begin{abstract}
The implementation of the National Oral Health Policy aimed to organize care networks in the Unified Health System. The investigation aims to evaluate the flow of dental services in the city of Fortaleza, Ceará, northeast of Brazil. This is a qualitative research, which used the Primary Health Care, Secondary (Center for Dental Specialties) and Tertiary Care. In the study, 04 managers and 09 dentists from the selected units participated. The collection of information, carried out during the year 2021, was extracted through an interview. The hermeneutic narrative was used to analyze the discourses, dividing them into: access to dental services, regulation of dental services and distribution of professionals in the network. It was found that the units have large territorial areas that generate geographic barriers to access the services offered. This is one of the factors that generate queues for procedures, causing significant absenteeism in the provision of services. It was also identified an insufficient number of professionals to meet the demand and failures in scheduling appointments. It was finished that it is necessary to create a standardized protocol for scheduling appointments in primary care, to expand the number of oral health professionals at this level of care and in dental specialty centers, and promote the implementation of new centers.
\end{abstract}

Keywords: Oral health; Health services accessibility; Appointments and schedules.

\section{Resumen}

La implementación de la Política Nacional de Salud Bucal buscó organizar redes de atención en el Sistema Único de Salud. La investigación tiene como objetivo evaluar el flujo de servicios dentales en la ciudad de Fortaleza, Ceará, noreste de Brasil. Se trata de una investigación cualitativa, que tuvo como escenario las unidades de Atención Primaria, Secundaria (Centro de Especialidades Odontológicas) y Terciaria de Atención de la ciudad. Participaron del estudio 04 gerentes y 09 odontólogos de las unidades seleccionadas. La recolección de información, realizada durante el año 2021, fue extraída a través de una entrevista. Se utilizó la narrativa hermenéutica para analizar los discursos, dividiéndolos en: acceso a los servicios odontológicos, regulación de los servicios odontológicos y distribución de los profesionales en la red. Se utilizó la narrativa hermenéutica para analizar los discursos, dividiéndolos en: acceso a los servicios odontológicos, regulación de los servicios odontológicos y distribución de los profesionales en la red. Se encontró que las unidades cuentan con grandes áreas territoriales que generan barreras geográficas para acceder a los 
servicios ofrecidos. Se encontró que las unidades cuentan con grandes áreas territoriales que generan barreras geográficas para acceder a los servicios ofrecidos. Este es uno de los factores que genera colas en los trámites, provocando un importante ausentismo en la prestación de los servicios. También identificó un número insuficiente de profesionales para atender la demanda y fallas en la programación de citas. Se concluyó que es necesario crear un protocolo estandarizado para la programación de citas en atención primaria, ampliar el número de profesionales de la salud bucal en este nivel de atención y en los centros de especialidades odontológicas, así como promover la implementación de nuevos centros.

Palabras clave: Salud bucal; Accesibilidad a los servicios de salud; Citas y horarios.

\section{Introdução}

A implantação da Política Nacional de Saúde Bucal (PNSB) procurou organizar uma estratégia de atenção à saúde que ainda estava invisível no âmbito do Sistema Único de Saúde (SUS) em meados de 2004. A PNSB pretendia fortalecer a atenção primária por meio das equipes de saúde bucal (ESB) e permitir o acesso a atenção especializada na odontologia por meio da implantação dos Centros de Especialidades Odontológicas (CEOs) e dos Laboratórios Regionais de Próteses Dentárias, e a viabilização da fluoretação das águas nas estações de tratamento de abastecimento público (Esposti et al., 2016), (Gabriel et al., 2020).

Várias avaliações realizadas pelo Ministério da Saúde demonstraram que indicadores positivos de saúde bucal foram favoráveis à diminuição das desigualdades em saúde após a implantação da PNSB no Brasil, apesar das dificuldades de regionalizar o serviço em todo o território nacional e ofertar atendimento público odontológico com amplitude universal. As equipes de Saúde Bucal dentro da Estratégia Saúde da Família (ESF) permitiram diminuir a incidência de cariogênese com a implementação das ações de promoção e prevenção em atividades coletivas e também intermediar um maior vínculo da população com as equipes (Gondinho et al., 2018), (Probst et al., 2019).

Os serviços odontológicos na saúde pública do país é uma necessidade indispensável, uma vez que $80 \%$ da população é dependente de cuidados dentários exclusivamente oferecidos pelo SUS (Probst et al., 2019). Desta forma, verifica-se que o aumento das equipes de saúde bucal na ESF pode viabilizar o acesso do usuário do SUS à consulta especializada por meio dos dispositivos de regulação assistencial, aumentando as chances da longitudinalidade do cuidado e finalização do tratamento odontológico (Galvão \& Roncalli, 2021).

Apesar dessa possibilidade, ainda persistem graves problemas de acesso universal à atenção à saúde bucal nas clínicas especializadas, os quais não são resolvidos, dentre eles: (1) atendimento à dor, trauma e infecções orais; (2) falhas no encaminhamento de casos ortodônticos, endodônticos e outros; e (3) tratamento restaurador atraumático (Nascimento et al., 2013).

No entanto, apesar da experiência exitosa da implantação da PNSB, verifica-se uma desestabilização do SUS, após a publicação da Emenda Constitucional n ${ }^{\circ}$ 95, que limitou o teto dos gastos com saúde e educação, a qual pode ter um impacto significativo em longo prazo no total dos investimentos destinados ao setor, em descompasso com as demandas da população. A portaria n 2.436 do Ministério da Saúde, que reformulou a Política Nacional de Atenção Básica (PNAB) em 2017, prevê a não obrigatoriedade da saúde bucal na ESF. O risco de possíveis retrocessos e de perda na qualidade dos serviços da atenção primária após a publicação dessa portaria é apontada como uma evidência (Lucena et al., 2020).

Nessa perspectiva, o objetivo dessa investigação é avaliar o fluxo de serviços da atenção odontológica no município de Fortaleza, Ceará, nordeste do Brasil. Ao mesmo tempo, procura entender como se operacionaliza a regulação desses serviços na rede de atenção primária, secundária e terciária desse município. 


\section{Metodologia}

Trata-se de uma pesquisa qualitativa, que demonstra o mapa organizacional dos fluxos e serviços da Rede de Saúde Bucal do Município de Fortaleza, Ceará. Utilizou-se como cenário para o estudo as unidades de Atenção Primária em Saúde (UAPS), secundária (Centro de Especialidades Odontológicas - CEOS) e terciária dos seguintes territórios: UAP Carlos Ribeiro; UAP Flávio Marcílio; UAP Anastácio Magalhães; UAP Luís Costa; UAP Graciliano Muniz; UAP Messejana; CEO Floresta e CEO Messejana. Além desses, foi investigado Serviço de Cirurgia e Traumatologia Bucomaxilofacial do Instituto José Frota (IJF), que oferta serviços na atenção terciária.

Quanto aos participantes do estudo, foram incluídos 04 gerentes e 09 dentistas das unidades selecionadas que tivesse pelo menos um ano de atuação na rede e fossem concursados. Foram excluídos participantes com menos de 1 ano de experiência no serviço odontológico; contratados e cooperados e que estivessem de licença ou férias.

A coleta de informações, realizada durante o ano de 2021, foi extraída por meio de uma entrevista. A entrevista foi realizada pelas pesquisadoras e dispunha de um instrumento que disparava os seguintes questionamentos: Como você analisa o acesso do usuário à consulta odontológica na atenção básica e especializada? Você considera rápida a marcação de consultas odontológicas do usuário para atenção especializada? Você consegue identificar as fragilidades e avanços na rede de saúde bucal do município?

Para analisar os discursos das entrevistas foi utilizada a narrativa hermenêutica, dividindo-se em fases as interpretações dos diálogos transcritos dos gestores e dentistas. Inicialmente foi elaborada uma primeira interpretação superficial dos textos, por meio de múltiplas leituras, no intuito de entender os temas principais que foram abordados. Em seguida, foi analisada a pré-compreensão, demonstrando como as interpretações se relacionam com o diálogo de autores que abordam esses conceitos. Entrevistas narrativas são entendidas como ferramentas não estruturadas, visando a profundidade, de aspectos específicos, a partir das quais emergem histórias de vida, tanto do entrevistado como as entrecruzadas no contexto situacional (Ricoeur, 1995). Os discursos foram divididos em: acesso aos serviços odontológicos; a regulação dos serviços odontológicos e distribuição dos profissionais na rede.

A interpretação em um discurso coloca-se entre a linguagem e a vida vivenciada por meio de uma série de conceitos interpretativos entre os quais há o distanciamento, a apropriação, a explicação e a compreensão. Um texto tem vários significados que podem ser interpretados diferentemente. O distanciamento não é imposto metodologicamente, ao contrário, é um aspecto constitutivo da sua transformação em texto escrito por meio da fixação da língua falada em língua escrita (Ricoeur, 1995).

O projeto de pesquisa foi submetido ao Comitê de Ética em Pesquisa da Universidade Estadual do Ceará, recebendo Parecer No 4.748.461, em 2021. Respeitou-se todos os preceitos éticos descritos na Resolução 466/2012 (Pinto et al., 2019).

\section{Resultados}

A Rede de Saúde Bucal de Fortaleza é constituída na atenção primária pelas UAPs que acolhe as demandas de grupos prioritários, faz a triagem das urgências e providencia encaminhamentos para o restante da rede.

A atenção secundária é representada pelos CEOS municipais e estaduais, pelas Unidades de Pronto Atendimento (UPAS), Urgência Odontológica da UFC, além das BB Clínicas. A atenção terciária é realizada em hospitais de grande porte como o IJF, atendendo desde lesões patológicas, trauma bucomaxilofacial e cirurgias ortognáticas, conforme Figura 1.

A investigação procurou analisar gestores da saúde bucal e odontólogos assistenciais verificando como ocorre o acesso à rede que perpassa ao longo da atenção primária e atenção secundária até chegar a terciária por meio da regulação. As falas dos entrevistados foram codificadas por G1, G2... referente aos gestores e dos cirurgiões dentistas em CD1, CD2... 
Figura 1. Mapa Organizacional da Rede de Saúde Bucal de Fortaleza, CE, Brasil, 2021.

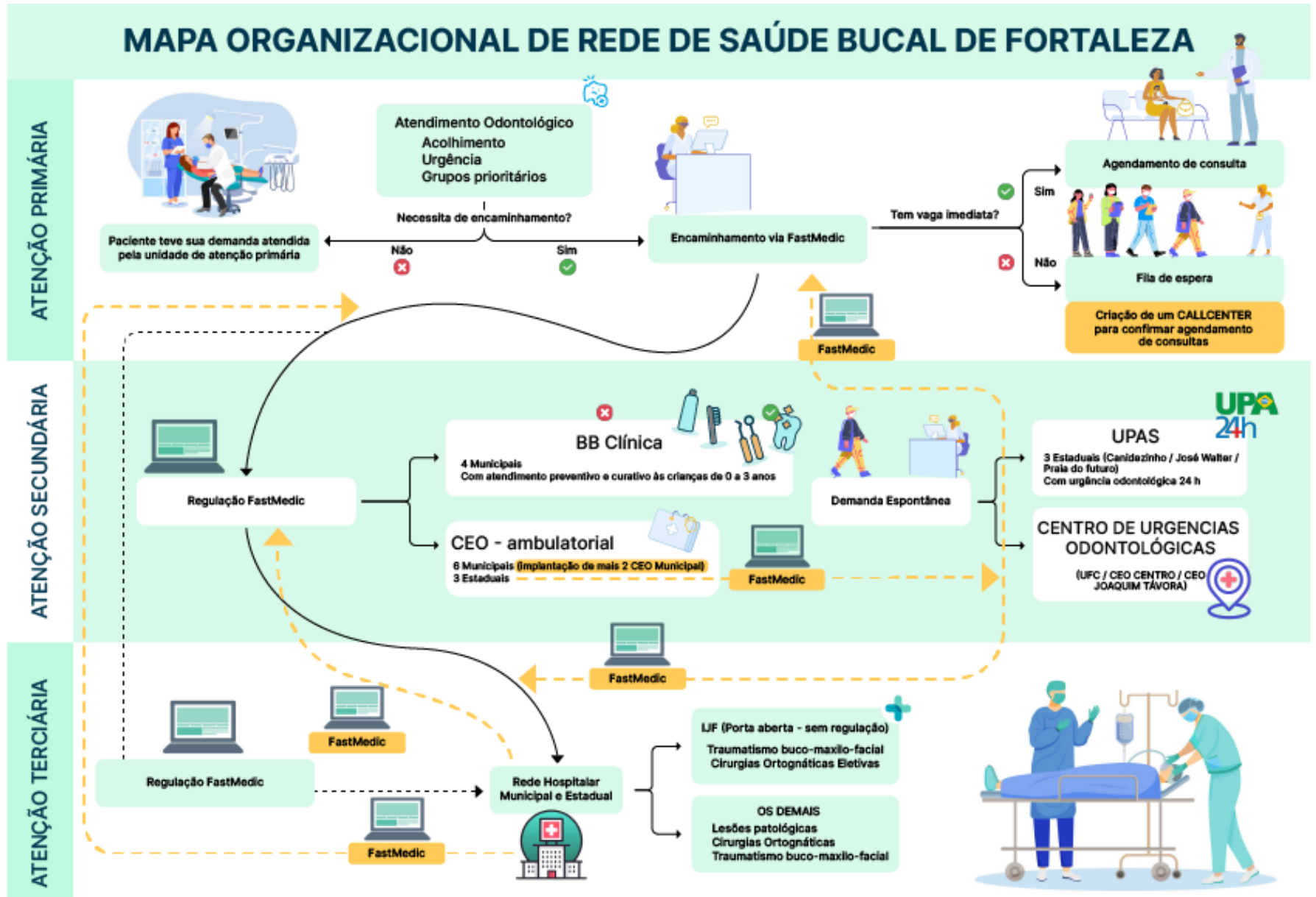

Fonte: Elaborado pelos autores.

\section{Acesso aos Serviços de Saúde Bucal no SUS}

Quando foi questionado aos gestores e cirurgiões dentistas como era o acesso de seus usuários às consultas odontológicas na atenção primária, no CEO e nos hospitais de referência, observaram-se os discursos:

[...] Existe uma população em torno de 38 a 39 mil habitantes e nós aqui somos três dentistas da Estratégia Saúde da Família. Então, é impossível que a gente consiga dar um fluxo rápido a demanda existente. Mesmo trabalhando a noite é impossível concluir o tratamento de todos os pacientes. Não há vagas suficiente para todos.... (CD1 - Atenção Primária).

[...] São filas que não andam, apesar de que agora tem uma central de regulação que eu acho que ajudou bastante. Mas, são filas muito extensas, né? ... e que faz o paciente desistir. O que é Endodontia vira Exodontia, o que era uma prótese parcial vira uma total e por aí vai... E ainda tem essas especialidades mais difíceis ainda de conseguir, né? Ainda tem as especialidades que tem a restrição da idade, como a ortodontia (CD3 - CEO).

[...] A gente tem inúmeros pacientes que perdem o procedimento, porque quando ele vai marcar, não há mais oferta. Às vezes ele espera tanto o procedimento que já não dá mais para fazer, entendeu? Devido essa espera, ele dá um jeito de fazer na rede privada, nos consultórios particulares... (CD7 - CEO). 
[...] Mas muitas vezes a gente escuta no consultório: " Doutora eu não tenho dinheiro, eu não tenho dinheiro para ir fazer a consulta". .... É uma realidade nossa! A gente ouve ele dizer que não vai à consulta no CEO porque o lugar onde mora fica muito distante e tem que pegar dois ou três transportes para se deslocar. Ou às vezes eles dizem que não vão para a consulta do território X ou Y porque a localidade onde fica o CEO é uma área de risco de violência (CD2 - Atenção Primária).

\section{A Regulação dos Serviços Odontológicos}

Em relação à opinião dos dentistas assistenciais e gestores das unidades de saúde bucal, foi possível verificar nas falas dos entrevistados como funcionava a regulação da rede que oferta consultas e procedimentos:

[...] Eu acho que a informatização melhorou bastante o acesso do usuário. Com o prontuário na unidade o dentista sabe quando o paciente veio se consultar. Isso foi um grande avanço! ...Na atenção secundária tínhamos apenas um CEO estadual. Mas apareceram outros com mais oferta de serviços.... Mas vejo como um ponto negativo o aumento da demanda, que está infinitamente maior a cada dia e isso prejudica o acesso a alguns procedimentos mais difíceis de marcar pelo Núcleo de Atendimento ao Cliente - NAC (CD 5 - CEO).

[...] Quando o NAC não dispõe do procedimento na hora da consulta, o paciente fica esperando três, quatro meses. Às vezes ele desiste de fazer o procedimento porque ele não vai vir aqui toda semana saber se seu procedimento foi agendado... (CD1 - Atenção Primária).

[...] A agilidade para marcar uma consulta depende muito da especialidade. Algumas consultas com especialistas são bastante demoradas para marcar, como a prótese, endodontia e ortodontia, que são extremamente difícil de se conseguir um agendamento. Outras, como a periodontia, ele consegue com um mês ou até dias... (CD6 - Atenção Primária).

\section{Distribuição dos Profissionais na Rede de Saúde Bucal}

Ao se questionar sobre as fragilidades e avanços da rede de saúde bucal no município de Fortaleza, o que mais se abordou foi a falta de profissionais odontólogos para suprir a grande demanda de atendimento, como se pode constatar por meio das falas:

[...] Eu vejo a necessidade gigantesca de se adquirir mais profissionais, por que a medida em que os serviços melhoram, as pessoas procuram mais o atendimento e cria-se uma grande demanda que sufoca, né? Eu acho que isso é um nó. Eu nem sei quantos profissionais a gente tem hoje na odontologia, mas sei que é bem menos do que há 10 anos para atender à população que temos atualmente (G2-CEO).

[...] Aqui há carência de profissional, certo? Então, para o usuário ter o acesso é bem complicado. No nosso posto temos apenas 3 dentistas para cobrir 8 áreas. Para o usuário começar a dar início a primeira consulta e também marcar encaminhamento para uma especialidade é bem difícil aqui. Há áreas descobertas por agentes de saúde.... (CD3 - Atenção Primária). 


\section{Discussão}

Após a apresentação da rede de serviços de saúde bucal com seu fluxo de atendimento, foi possível identificar fatores que impedem o usuário de caminhar por essa rede no município de Fortaleza. Verificou-se que as unidades de saúde se encontram em áreas territoriais de grande dimensão que geram barreiras geográficas para se ter acesso aos serviços especializados oferecidos, também impedindo a comunicação entre o fluxo na rede. Esses são fatores que provocam filas de espera por procedimentos e consultas, ocasionando um absenteísmo muito significativo na execução dos atendimentos.

Investigações anteriores relacionadas ao acesso aos serviços odontológicos no SUS revelaram que há uma insatisfação do usuário relacionada ao tempo de espera e ao tempo necessário para resolver a demanda. Em nível individual, o tempo de espera elevado e a não conclusão do tratamento aumentam as chances de insatisfação dos usuários com o serviço de assistência odontológica. É importante que usuários sejam capazes de romper com o sistema hegemônico de atenção à saúde do país, este, centrado na assistência curativa, especializada e hospitalar, passando a utilizar preferencialmente práticas promotoras e preventivas de saúde oferecidas pelas equipes da Atenção Primária (Gonçalves et al., 2021).

Lucena et al. (2020) argumenta que a redução na oferta de serviços de saúde bucal nos locais mais desiguais poderá impactar de forma negativa o acesso aos serviços odontológicos, bem como atividades individuais e coletivas. O cenário político econômico atual aponta para a acentuação das desigualdades e das iniquidades em saúde, o que representa um quadro sombrio para população brasileira dependente do SUS (Borges et al., 2016), (Lucena et al., 2020). Essas iniquidades encontradas entre os municípios brasileiros, em sua maioria de pequeno porte populacional, intensifica a fragmentação do sistema e provoca disputas por recursos federais, dificultando a conformação das redes intermunicipais e adia o processo de regionalização do sistema de saúde brasileiro em sua capacidade resolutiva (Esposti et al. 2016), (Godoi et al. 2020).

A capacidade dos profissionais das equipes de saúde para convencer os usuários sobre a importância de completar seu tratamento, a possibilidade de realização de busca ativa pelos agentes comunitários de saúde e a garantia de reconsulta parecem contribuir para uma frequência maior de tratamentos odontológicos concluídos. Fatores como a baixa condição social, a dificuldade de acesso, a falta de informação sobre saúde bucal, sua manutenção e tratamento, a desmotivação ou o medo do tratamento, o relacionamento do paciente com a equipe de profissionais pode interferir na conclusão desse tratamento (Viana et al., 2019).

Para a organizar o acesso dos serviços odontológicos na atenção primária, faz-se necessário que os profissionais de saúde estejam envolvidos no processo de primeira escuta. Desta forma, devem estar atentos às necessidades de saúde bucal do usuário e ter ciência tanto da classificação de risco das urgências odontológicas, quanto do fluxo de encaminhamento desse usuário para a equipe de saúde bucal. Para que o acolhimento às situações de saúde bucal possa ocorrer adequadamente, é fundamental que atuem, em conjunto, todos os profissionais da unidade, bem como os apoiadores e os gestores, os trabalhadores da regulação (Basso et al., 2019). Souza et al. (2015) pontua que existem falhas no uso da referência e contra referência entre a atenção primária, secundária e terciária que fazem o usuário ter dificuldade de acesso a procedimentos que são encaminhados no percurso da rede.

O Brasil Sorridente, como modelo de reorientação das práticas em saúde bucal no SUS, visa ampliar a atenção à saúde bucal e o enfrentamento contra a cultura do odontocentrismo e da extração-mutilação dentária que permeia o imaginário odontológico, tanto subjetivo quanto midiático. Trata-se de desafiadora proposta, uma vez que as teorias odontológicas hegemônicas reproduzem esse centramento dentário. No imaginário odontológico da população, a extração dental é um mediador para a retirada da dor, resolvendo o problema a um custo operacional muito baixo (Esposti et al. 2016).

Dentre as falhas do fluxo de encaminhamento e agendamento de consultas/ procedimentos é possível considerar a presença de muitas áreas descobertas por agentes de saúde e ausência de um protocolo padrão com a finalidade de avaliar as 
consultas especializadas. A consulta, na maioria das vezes, é agendada sem critério ou classificação de risco odontológico, ou seja, inexiste um canal de comunicação entre serviço e usuário ou não há a avaliação do perfil clínico do paciente.

Sobre a organização dos serviços odontológicos, é importante entender que a realização de procedimentos de atenção básica nos CEOs deve ser restringida aos pacientes com necessidades especiais, uma vez que este serviço tem custo mais elevado quando é realizado em um serviço especializado. Por um lado, espera-se que os CEOs restrinjam a oferta de procedimentos de atenção básica de livre demanda (Andrade et al., 2020).

O tratamento especializado continua sendo de difícil acesso dentro do SUS. A atenção primária deve resolver coisas simples, tais como: problema de gengivite, fazer limpeza nos dentes e avaliações periódicos de procedimentos rotineiros (Fonseca et al., 2015). No entanto, é importante investir de forma mais enérgica no CEO em especialidades básicas (periodontia, endodontia e cirurgia) para fortalecer o cuidado bucal da população em municípios mais carentes desses serviços (Lima \& O'dwyer, 2020).

Estudos já realizados nos estados do Amazonas, Maranhão, Bahia e Paraíba sobre o acesso às consultas especializadas nos CEOs demonstraram insatisfação dos usuários com o atendimento, o que ocasiona um baixo desempenho de metas estabelecidas pelo Ministério da Saúde na avaliação dos serviços odontológicos (Galvão \& Roncalli, 2021).

No tocante ao funcionamento da regulação, os entrevistados expuseram que a informatização desse setor facilitou a agilidade na marcação de consultas. Mesmo assim, ainda há uma grande fila de espera por procedimentos especializados que impedem a conclusão dos tratamentos odontológicos.

Os resultados de uma investigação na gestão da saúde bucal realizada no Estado de Santa Catarina apontam que a atual discussão sobre implantação do complexo regulador ambulatorial pode influenciar na estruturação de uma rede regionalizada de atenção à saúde bucal. Há estímulos para envolvimento de coordenadores da atenção à saúde bucal nas discussões para iniciar a identificação dos serviços, especialmente os relacionados à Rede da Pessoa com Deficiência, e para construção de fluxos e protocolos para regulação. Apesar de ser reconhecida a grande expansão de serviços de atenção básica também relacionados à saúde bucal, a escassez de serviços especializados é constatada da mesma forma. A ausência de protocolos, diretrizes e regulação via Central de Regulação Ambulatorial são falhas na Transparência, impactam nas relações de Accountability e foram apontadas como razões para a dificuldade de integração entre as ações de saúde bucal e provisão de serviços odontológicos (Godoi et al. 2020).

As centrais de regulação devem ofertar agendamento rápido principalmente aos grupos prioritários ao atendimento odontológico, como as pessoas com deficiência. Um estudo já realizado no município de Fortaleza demonstrou que aproximadamente um terço das pessoas com deficiência afirmaram nunca ter procurado atendimento odontológico nas UAPs e cerca de $40 \%$ nunca foram examinados por dentista no serviço público de saúde do município. A causa dessa lacuna de serviço não é clara, mas pode ser baseada nos perfis sociodemográficos, educacionais e culturais das pessoas com deficiência, a falta de prioridade dada aos serviços para deficientes, incompreensões do sistema de atendimento odontológico e barreiras à comunicação e acesso aos serviços de saúde causados por problemas geográficos e arquitetônicos na área local e nas UAPs (Rocha, Saintrain \& Vieira-Meyer, 2016).

Outras investigações que analisaram o agendamento da consulta especializada apontam que os pacientes esperam 3 a 4 meses para realizar um tratamento endodôntico principalmente quando existe a presença de dor. Um período de 5 meses de espera por um tratamento endodôntico, como encontrado neste estudo, pode gerar várias consequências negativas como a busca constante do paciente por tratamentos de urgência com intuito de alívio da dor, sobrecarregando a atenção primária com esta demanda, além da possibilidade de ocorrer fratura do elemento dental e/ou desistência do paciente pelo tratamento. Estes dois últimos fatores podem resultar na exodontia do elemento dentário (Magalhães et al., 2019). 
Os entrevistados dessa pesquisa disseram ainda que o número de profissionais da saúde bucal é insuficiente para dar resposta à grande demanda que procura atendimento odontológico. Sobre esse fato, pode-se inferir que embora os postos de trabalho no SUS tenham aumentado, pesquisas ainda apontam uma tendência crescente na má distribuição dos dentistas no país, principalmente por considerarem que as concentrações das instituições formadoras e o melhor poder aquisitivo da população nos grandes centros influenciam na decisão do lugar de atuação profissional, já que esta classe busca uma dupla prática público/privada. A falta de um plano de cargos e salários pode colaborar com esse comportamento e favorecer a rotatividade dos cirurgiões dentistas no SUS (Gabriel et al., 2020).

O quantitativo de ESB implantadas nos municípios brasileiros reduziu após a PNAB 2017. Verificou-se maior redução nos municípios localizados nas regiões Nordeste e Sul, com maior porte populacional. O número de municípios que reduziram a quantidade de ESB após um ano e nove meses da publicação da PNAB 2017 triplicou (Lucena et al., 2020). Desta forma, é necessária que a PNSB seja reformulada e fortalecida pelo Ministério da Saúde e demais entes federados.

\section{Conclusão}

A investigação identificou falhas nos fluxos da rede de atenção primária, secundária e terciária da saúde bucal de Fortaleza. Sendo assim, foram relatados pelos entrevistados que há filas na espera por atendimentos especializados, um número de profissionais insuficientes para suprir a demanda e falhas na marcação de consultas.

Dessa forma, faz-se necessário criar um protocolo padronizado de marcação de consultas na atenção primária para que seja efetivado o agendamento das consultas odontológicas especializada; criação de um call center em todas as unidades de saúde para que haja um canal direto com o usuário nas confirmações dos seus agendamentos; operacionalizar aplicativos ou dispositivos móveis para que o usuário visualize o agendamento de sua consulta pelo celular ou outros meios; distribuir os sistemas de regulação (fastmedic) nos hospitais terciários, UPAS e CEOs estaduais para fortalecer a referência e contra referência; ampliar o quadro de profissionais na saúde bucal da atenção primária e nos centros de especialidades odontológicas, bem como promover a implantação de novos centros.

Novas pesquisas devem ser desenvolvidas para verificar também qual o absenteísmo gerado na rede de serviços da regulação odontológica do município de Fortaleza.

\section{Referências}

Andrade, F. B., Pinto, R. S. \& Antunes, J. L. F. (2020). Tendências nos indicadores de desempenho e monitoramento de produção dos Centros de Especialidades Odontológicas do Brasil. Cadernos de Saúde Pública. 36(9):e00162019.

Basso, M. B., Nunes, N. B., Corrêa, L. B. C., Vieira, C. N., Vilarinho, J. L. P. S. \& Pucca Júnior, G. A. (2019). A construção da rede de atenção à saúde bucal no Distrito Federal, Brasil. Ciência \& Saúde Coletiva. 24(6):2155-2165.

Borges, T. S., Schwanke, N. L., Reuter, C. P., Neto, L. K., \& Burgos, M. S. (2016). Factors associated with caries: a survey of students from southern Brazil. Revista Paulista de Pediatria. 34(4):489-494.

Esposti, C. D. D., Cavaca, A. G., Côco, L. S. A., Santos-Neto, E. T. \& Oliveira, A. E. (2016). As dimensões do acesso aos serviços de saúde bucal na mídia impressa. Saúde e Sociedade. 25(1):19-30.

Fonseca, L. L. V., Nehmy, R. M. Q. \& Mota, J. A. C. (2015). O valor social dos dentes e o acesso aos serviços odontológicos. Ciência \& Saúde Coletiva. 20(10):3129-3138.

Gabriel, M., Cayetano, M. H., Chagas, M. M., Araújo, M. E., Dussault, G., Pucca Júnior, G. A. \& Almeida, F. C. S. (2020). Mecanismos de ingresso de dentistas no SUS: uma agenda prioritária para o fortalecimento do Brasil Sorridente. Ciência \& Saúde Coletiva. 25(3):859-868.

Galvão, M. H. R. \& Roncalli, A. G. (2021). Desempenho dos municípios brasileiros quanto à oferta de serviços especializados em saúde bucal. Cadernos de Saúde Pública.37(1):e00184119. 
Godoi, H., Castro, R. G., Santos, J. L. G., Moyses, S. J. \& Melo, A. L. S. F. (2020). Óbices da governança pública e sua influência sobre a atenção à saúde bucal no Estado de Santa Catarina, Brasil. Cadernos de Saúde Pública. 36(11): e00184719.

Gonçalves, K. F., Menegazzo, G. R., Hilgert, J. B., Hugo, F. N., \& Giordani, J. M. A. (2021). Fatores contextuais e individuais associados à insatisfação com a assistência odontológica no Brasil. Ciência \& Saúde Coletiva. 26(2):3715-3724.

Gondinho, B. V. C., Guerra, L M., Bulgareli, J. V., Probst, L. F., Cortellazzi, K. L., Possobon, R. F., Pereira, A. C., \& Oliveira, V. A. S. (2018). Percepção de coordenadores de saúde bucal sobre a rede de atenção à saúde bucal. Revista Brasileira em Promoção da Saúde. 31(Supl): 1-8.

Lima, F. L. T. \& O’Dwyer, G. (2020). Políticas de Prevenção e Controle do Câncer Bucal à luz da Teoria da Estruturação de Giddens. Ciência \& Saúde Coletiva. 25(8):3201-3214.

Lucena, E. H. G., Lucena, C. D. R. X., Alemán, J. A. S., Pucca Júnior, G. A., Pereira, A. C. \& Cavalcanti, Y. W. (2020). Monitoring of oral health teams after National Primary Care Policy 2017. Revista de Saúde Pública. 54:99.

Magalhães, M. B. P., Oliveira, D. V., Lima, R. F., Ferreira, E. F., \& Martins, R. C. (2019). Avaliação da atenção secundária em endodontia em um Centro de Especialidades Odontológicas (CEO). Ciência \& Saúde Coletiva. 24(12):4643-4654.

Nascimento, A. C., Moysés, S. T., Werneck, R. I., \& Moysés, S. J. (2013). Oral health in the context of primary care in Brazil. Int Dent J. 2013 Oct;63(5):23743.

Pinto, J. R., Pedrosa, K. A., Martins, P., Ferreira Júnior, A. R. \& Maranhão, B. D. R. (2019). (Dis) connections between health councils and audit: advancements and challenges in the democratization of public health management. Cadernos Saúde Coletiva. 27(1):39-44.

Probst, L. F., Pucca Júnior, G. A., Pereira, A. C., \& Carli, A. D. D. (2019). Impacto das crises financeiras sobre os indicadores de saúde bucal: revisão integrativa da literatura. Ciência \& Saúde Coletiva. 24(12): 4437-4448.

Ricoeur, P. (1995). Hermeneutics and the human sciences. Cambridge: Cambridge University Press.

Rocha, L. L., Saintrain, M. V. L. \& Vieira-Meyer, A. P. G. F. (2015). Access to dental public services by disabled persons. BMC Oral Health. 15(35): 3-9.

Souza, G. C. S. A., Lopes, M. L. D. S., Roncalli, A. G., Medeiros-Júnior, A. \& Clara-Costa, I. C. (2015). Referência e contra referência em saúde bucal: regulação do acesso aos centros de especialidades odontológicas. Revista de Salud Pública. 17(3):416.

Viana, I. B., Moreira, R. S., Martelli, P. J. L., Oliveira, A. L. S., \& Monteiro, I. S. (2019). Avaliação da qualidade da assistência em saúde bucal na Atenção Primária à Saúde em Pernambuco, 2014. Epidemiologia e Serviços de Saúde. 28(2):e2018060. 